\title{
Compensation Rigging by Powerful CEOs: A Reply and Cross-Sectional Evidence* $^{*}$
}

\author{
Adair Morse $^{1}$, Vikram Nanda ${ }^{2}$ and Amit Seru ${ }^{3}$ \\ ${ }^{1}$ Haas School of Business, University of California, Berkeley \\ ${ }^{2}$ Rutgers Business School \\ ${ }^{3}$ Booth School of Business, University of Chicago
}

\begin{abstract}
Wan (2013) argues that the statistical inferences in our Journal of Finance (2011) paper are not robust, as we do not prove that it is powerful CEOs that rig incentive contracts. Wan makes the theoretical claim that the rigging results are consistent with ex-post optimal re-contracting. However, optimal re-contracting cannot explain the loss in firm value from contract switching we show in the paper. Nor do we know of a theory that would predict that ex-post realignment could be tested using our contract switching term in the wage function, like Wan does. On the empirical front, Wan's critique has at least three flaws. First, his standardized performance measures - different than ours - result in accounting returns being 14 percentage points higher than stock returns. Consequently, switching between measures, necessary for identification, is infrequent and outlier-based, not surprisingly delivering regression estimates different from ours. Second, he interprets selectively among insignificant coefficients to make his claims. Third, regardless of interpretation, basic mathematics casts doubt on the premise of his estimation strategy. Wan makes one valid point: our original work could have provided more extensive cross-sectional empirical support for our rigging claims. We take this opportunity to present new cross-sectional (between-firm) evidence and conclude even more strongly that powerful CEOs sway boards to load their incentive pay on more favorably performing measures.
\end{abstract}

* We thank the editor (Ivo Welch) for helpful comments.

ISSN 2164-5744; DOI 10.1561/104.00000019

(C) 2013 A. Morse, V. Nanda and A. Seru 
Keywords: $\quad$ CEO compensation; rigging; corporate governance.

JEL Codes: $\quad$ G34, J31, J33

In our Journal of Finance (2011) paper (hereafter MNS), we argue that powerful CEOs induce their boards to shift the weight on performance measures towards measures that are ex-post more favorable; thereby rigging the incentive part of their pay. The intuition is developed in a simple model in which some powerful CEOs exploit superior information and lack of transparency in compensation contracts to extract rents. The model delivers an explicit structural form for the rigging of CEO incentive pay along with testable implications that rigging is expected to: (1) increase with CEO power, (2) increase with CEO human capital intensity and uncertainty about a firm's future prospects, and (3) negatively impact firm performance. We then test this theory by estimating a standard CEO compensation equation as a function of performance indicators and economic variables with an additional term that captures rigging by powerful CEOs. In line with the wage equation predicted by our theory, we include a variable, Max *Power, that captures the return of the ex-post performance measure that does relatively better that period $(M a x),{ }^{1}$ interacted with a measure of CEO power (Power). Using various measures of CEO power and board independence in a large panel of firms in the U.S., we find support for our predictions in specifications that identify off time-series changes within firms.

Wan (2013) affirms our baseline regression results on rigging. However, he argues that since our main estimation equation does not include the uninteracted term $M a x$, our results are biased. ${ }^{2}$ He contends that our results

1 Max represents the better performance measure in that period when choosing between standardized stock returns and standardized accounting returns.

2 We had originally included the Max term in earlier drafts of our published paper. We had dropped this term in the reviewing process where we were asked to conform the estimated wage function to that predicted by our theory. We reproduce results from the earlier drafts, with both Max and Max* Power terms in the appendix. The coefficient on the interaction term survives for most measures of power variables even when we include Max. Notably, there is no specification where we find Max is significant and positive while $M a x *$ Power is not. We relegated this comment to a footnote because, given the lack of significance on Max and $\operatorname{Max} *$ Power for some power variables — as we will discuss, due to high multi collinearity - these estimations may not entirely convince readers that rigging is being driven by CEOs with power across all measures. This is a task that our new cross sectional tests are better equipped to demonstrate. 
could be explained by optimal re-contracting (for example, repricing of executive options). We take the task of robustness to Wan's claim of omitted variable bias seriously. It is a fair question to ask whether rigging happens only in the powerful-CEO firm years. We strongly reconfirm our results here, providing new tests that exploit cross sectional variation, that rigging only occurs in firms where CEOs have power. In doing so, we argue that Wan's critique is not supported theoretically or empirically.

In Wan's estimations that include both Max and Max * Power - using his standardized performance measures, which differ from ours as described below - neither coefficient is significant. Wan interprets this to imply that only Max matters. Beyond this rather curious choice as to which insignificant coefficient can be interpreted, we do not understand why or how optimal ex-post incentive alignment (which Wan contends can explain our finding) should result in a wage function with a contract switching term like ours (Max). Furthermore, an "optimal contract" with Max would be difficult to reconcile with the lower value/performance that MNS find to be associated with rigging. We discuss the theoretical problems with Wan's proposed alternative in some detail.

But, irrespective of the theoretical inconsistency, Wan cannot estimate both terms in a fixed effects model with high correlation (over 0.9) between the two variables in his data. In particular, the fact that inclusion of Max drives down the statistical significance of both Max and Max*Power - rendering both insignificant - is not due to an omitted variable, as Wan claims, but rather due to high multicollinearity between Max and Max * Power. We derive the mathematics hindering this estimation and explain that cross sectional tests can help distinguish firms where rigging occurs.

Next, we report evidence from tests that exploit cross sectional variation to isolate where rigging occurs. We find strong evidence that rigging only occurs in powerful-CEO firms by conducting a series of tests which assess sensitivity of compensation to Max using power level interactions, using stratification of the sample by power (sorts), and using a between-effects estimator. The integrity and empirical robustness of power-induced rigging is confirmed even more strongly in this analysis.

We have two other points, which are more rebuttal in nature. The first issue involves the construction of the Max variable. Wan's standardization of the performance measures differs from ours. We standardize accounting and stock returns to be mean zero in the data. The idea is that accounting and stock returns have very different distributions; thus one cannot compare 
a good performance (for paying compensation) in terms of accounting returns relative to stock returns without making them comparable in distribution. Wan agrees, but standardizes the measure in the larger set of firms in ExecuComp (ExecuComp backfilled a larger sample, mostly smaller firms, after we completed our paper). Under Wan's standardization, he is taking a maximum over two returns measures with accounting returns having a mean of +0.070 and stock returns having a mean of -0.074 , a 14 percentage points difference. Wan's taking a Max of these measures means the maximum loads systematically on accounting returns, in particular in two-thirds of the cases. This pattern substantially differs from the construction of Max from our sample. Five implications emerge.

The first implication is that Wan's results differ from ours because his Max only picks up stock returns in extreme cases of very high stock returns or very low accounting returns. Thus, it is hard to interpret his tests as a direct replication of the MNS paper or its theory. Relatedly, given that Wan's measure loads up largely on accounting performance, it is also not surprising that his analysis only picks up rigging with some asymmetry. Second, his construction of Max eliminates half of the cases of switching needed to identify the coefficient of interest in a fixed effects estimation. Thus, it is quite surprising that Wan finds any, even weaker significance across the board. The third implication is that Wan's method accentuates the multi collinearity concern, reinforcing the need to use cross sectional tests to identify where rigging occurs.

Fourth, Wan finds that introduction of interactions of Max with indicators for low accounting and low stock performance render Max insignificant, though its interaction with low stock performance is significant. Wan claims that this evidence is proof of option repricing. Leaving aside the issue that this claim is unsubstantiated, we show that these findings are, once again, an artifact of the way in which Wan has constructed his Max variable. In fact, when using our measure, we find Max interaction with low accounting performance to be significant as well.

Finally, Wan writes that our results are not robust when returns are not winsorized. In fact, as we show in this paper (and so does Wan), our results are quite robust to not winsorizing. The fact that some of Wan's results are sensitive to not winsorizing is again attributable to how he constructs his Max: the only observations which identify coefficients on his Max and his Max*Power are extremely high stock returns and extremely low accounting returns - ones which are directly impacted by his winsorization. 
The second rebuttal concerns his claim that our rigging results are solely driven by new, externally-hired CEOs. Although we are interested in these CEOs as well, we find rigging in pay by powerful CEOs even when we remove externally-hired CEOs. The reason for discrepancy is that Wan's "new" externally-hired CEOs are actually, by his definition, CEOs who were hired from outside the firm. His result appears to have nothing to do with these executives being new CEOs as such, but rather being CEOs who at some point in time were hired externally. Regardless, in ExecuComp data with our measure of Max, newly hired CEOs rig their pay when they have power - a result consistent with MNS.

The contribution of this note is twofold in nature. As a rebuttal, we explain why Wan's critique is not supported theoretically or empirically. More importantly perhaps, the opportunity to write this note has allowed us to explore rigging from a cross sectional angle, which we did not do as extensively in our original paper. We conclude even more strongly now that powerful CEOs indeed seem to sway boards to load their incentive pay on more favorably performing measures.

\section{Theory: Starting Points of MNS and Wan}

Wan argues that, since our main specifications do not include the level term Max, our results are biased. We begin refuting this claim by conveying why the baseline specification in MNS was chosen, in a brief overview of our theory. We then comment on Wan's derivation of the empirical bias.

\subsection{Rigging Theory}

MNS use a familiar one-period contracting environment in which a riskaverse CEO provides costly but unobservable effort, and two performance measures provide noisy indications of the CEO's effort level. We allow for a state variable that is observed by the board, though not by outsiders. Independent boards offer the CEO an optimal contract conditional on the state. The set of possible contracts that could be offered by the independent boards forms the complete set of contracts that shareholders expect to see in equilibrium and, thereby, limits the contract choices of a compromised board as well. ${ }^{3}$ Despite the pooling in compensation arrangements, we show that

3 Specifically, in the model, board members derive benefits from their position and avoid actions that may precipitate their removal. We assume that boards that are revealed to be compromised trigger 
asymmetric information and the lack of transparency in CEO compensation arrangements can allow some powerful CEOs to rig their compensation without triggering outside shareholder outrage a la Bebchuk et al. (2002). Specifically, the flexibility provided to the compromised board in the form of multiple performance measures allows some powerful CEOs to use ex-post information to extract rents by shifting the weight on performance measures towards the ones with a relatively more favorable outcome.

Our model delivers the following CEO wage function, under the assumption that the shifting of weights on performance measures towards the favorable one is symmetric for the two measures:

$$
\mathrm{Y}_{i t}=\left\{\begin{array}{c}
\beta_{1} \text { Power }_{i t}+\beta_{2} z \text { RStock } \\
+\beta_{p} \text { Power }_{i t} * z R S \beta_{3} z R O \text { to }_{i t} * I_{z R O S>z R O A} \\
+\beta_{p} \text { Power }_{i t} * z R O A_{i t} * I_{z R O A>z R O S}+\eta_{i t}
\end{array}\right\} .
$$

In this equation, $z R S t o c k$ and $z R O A$ are stock and accounting performance measures that are normalized by using industry mean and volatility to make them directly comparable. $I$ is a dummy variable that takes a value 1 if one performance measure is ex-post doing better than the other and 0 otherwise. Rigging by powerful CEOs implies that $\beta_{p}>0$. Combining the two interaction terms gives us the CEO wage function in which the incentive portion consists of a rigging term that increases with CEO power:

$$
\begin{aligned}
Y_{i t}= & \beta_{1} \text { Power }_{i t}+\beta_{2} z \text { RStock } \\
& +\beta_{p} \text { Power }_{i t} * \beta_{3} z R O A_{i t} \\
& \operatorname{ax}\left\{z \text { ROS }_{i t}, z R O A_{i t}\right\}+\eta_{i t}
\end{aligned}
$$

This is the equation that we estimate in MNS. The strong tie between the wage equation we test and the model also reflects the culmination of the refereeing process at the Journal of Finance.

Wan (2013) suggests that the equation may be misspecified since it does not include the variable $\operatorname{Max}\left\{z R O S_{i t}, z R O A_{i t}\right\}$, that is, Max not interacted with Power. Observe that including this variable is equivalent to the inclusion of two additional variables, $z R S t o c k_{i t} * I_{z R O S>z R O A}$ and

shareholder outrage, leading to their eventual ouster through a proxy fight or by takeover. Hence, in equilibrium, compromised boards have the incentive to mimic the actions of independent boards and avoid revealing their type. We analyze such a pooling equilibrium - specifically, one in which all boards pool by offering similar types of compensation. Since independent boards have no reason to deviate from offering contracts they consider optimal, the only contracts observed in the pooling equilibrium will be ones that an independent board might choose. 
$z R O A_{i t} * I_{z R O A>z R O S}$. Neither our model nor Wan's discussion delivers insights into how these variables could reflect an optimal contract for all firms in equilibrium, as Wan suggests. As noted, the value and performance loss associated with rigging that we document in the paper is hard to reconcile with optimal contracting.

\subsection{Wan's Derivation of Bias}

Instead of providing an economic basis for assuming that optimal contract changes ex-post should occur in precisely the form derived in our model ( $\operatorname{Max}$ ), Wan derives the potential bias from omitting Max in our baseline specification by writing down an equation that CEO Power and Max*Power cause Max:

$$
\operatorname{Max}_{i t}=b_{0}+b_{1} \text { Power }_{i t}+b_{2} \text { Max }_{i t} * \text { Power }_{i t}+\delta_{i t} \text {. }
$$

Wan plugs the predicted value from this equation into the main wage estimating equation and derives how our estimates must be biased. In fact, this equation is supportive of the MNS hypothesis; CEO power causes rigging since in Wan's equation the variation in Max is driven by Power and Max * Power. However, the idea that an interaction term (Max* Power) could be an explanatory variable for the level term from which it is derived (Max) does not make economic sense to us. While we do not understand the specification, we are fine with the result that power is causing rigging.

Although Wan did not make his arguments along these lines, in order to show there was an omitted variable bias he could have investigated whether the residual from his (first-stage) equation above was significant when included in the CEO compensation estimation Equation (1). We perform this exercise in Table 1. In columns 1-3 we present Wan's equation projecting Max on Power and Max*Power. As can be observed from columns 4-6, the corresponding residuals from the three regressions presented in columns 1-3 have no explanatory power in the CEO compensation equation for all the measures of power. Importantly, the coefficients on Max* Power are unaffected. While we do not advocate Wan's equation or his approach, this analysis does clarify that the omitted variable bias that Wan is concerned about is not at play.

We think that Wan's starting his arguments with this empirical derivation of bias, which he never actually explores, is not a basis for asserting that 


\begin{tabular}{|c|c|c|c|c|c|c|}
\hline & \multicolumn{3}{|c|}{ Dependent Variable: $\operatorname{Max}$} & \multicolumn{3}{|c|}{ Dependent Variable: $\operatorname{Ln}(T D C)$} \\
\hline & (1) & (2) & (3) & (4) & (5) & (6) \\
\hline $\begin{array}{l}\text { Max } * \text { Power } \\
\quad \text { Index }_{i t}\end{array}$ & $\begin{array}{l}0.434^{* * *} \\
{[0.004]}\end{array}$ & & & $\begin{array}{c}0.028^{* *} \\
{[0.012]}\end{array}$ & & \\
\hline Max*Insider $\%_{i t}$ & & $\begin{array}{l}2.671^{* * *} \\
{[0.080]}\end{array}$ & & & $\begin{array}{c}0.233^{* * *} \\
{[0.077]}\end{array}$ & \\
\hline $\begin{array}{l}\text { Max } \%^{*} \\
\quad \text { Appointed }_{i t}\end{array}$ & & & $\begin{array}{l}1.134^{* * *} \\
{[0.013]}\end{array}$ & & & $\begin{array}{c}0.090^{* * *} \\
{[0.033]}\end{array}$ \\
\hline $\operatorname{Residual}_{i t}^{\text {Power }}$ & & & & $\begin{array}{c}0.022 \\
{[0.048]}\end{array}$ & & \\
\hline Residual $_{i t}^{\text {Insider }}$ & & & & & $\begin{array}{c}0.027 \\
{[0.034]}\end{array}$ & \\
\hline Residual $_{i t}^{\text {Appointed }}$ & & & & & & $\begin{array}{c}0.045 \\
{[0.039]}\end{array}$ \\
\hline Power Index & $\begin{array}{c}-0.157^{* * *} \\
{[0.008]}\end{array}$ & & & $\begin{array}{r}0.0488 * \\
{[0.0258]}\end{array}$ & & \\
\hline Insider $\%_{i t}$ & & $\begin{array}{c}-1.116^{* * *} \\
{[0.102]}\end{array}$ & & & $\begin{array}{c}0.0033 \\
{[0.0605]}\end{array}$ & \\
\hline$\%$ Appointed $_{i t}$ & & & $\begin{array}{c}-0.401^{* * *} \\
{[0.029]}\end{array}$ & & & $\begin{array}{c}-0.0696 \\
{[0.0928]}\end{array}$ \\
\hline Observations & 10231 & 7551 & 7004 & 8263 & 6121 & 5797 \\
\hline $\mathrm{R}^{2}$ & 0.861 & 0.606 & 0.625 & 0.239 & 0.219 & 0.221 \\
\hline $\begin{array}{l}\text { Time \& } \\
\text { Firm F.E. }\end{array}$ & Yes & Yes & Yes & Yes & Yes & Yes \\
\hline Other Controls & Yes & Yes & Yes & Yes & Yes & Yes \\
\hline
\end{tabular}

Note: ${ }^{* * *},{ }^{* *}$ and $*$ denote significance at $1 \%, 5 \%$ and $10 \%$ respectively.

Table 1. Omitted variable bias based on projection equation in Wan (2013).

Description: In this table we report the estimates from the projection regression of Max on Power and Max $*$ Power as suggested in Wan (2013). We iteratively employ the three power measures PowerIndex, Insider\% and \%Appointed-in a firm fixed effects specification. The residuals from the projection equation are then included in the last three columns to account for the 'omitted variable' problem that worries Wan (2013). The last three regressions use the natural log of the totalcompensation of the CEO as the dependent variable. Controls employed are same as in MNS,including performance and lagged performance. All regressions are estimated with time effects, and robust standard errors are reported in the parenthesis. Data in this table is for the period 1992 to 2003.

Interpretation: Omitted variable bias would imply that the residuals from the projection of Max on Power and Max*Power (columns 1-3) would be significant when included in the CEO compensation estimation equation. The residuals corresponding to the regressions in columns 1-3 have no explanatory power in the CEO compensation equation (columns 4-6) and the coefficients on Max * Power are unaffected. This suggests absence of omitted variable bias that Wan is worried about. 
omitted bias must be important. In fact we just showed it is not. Wan connects his derivation to his finding that the inclusion of Max drives out the statistical significance of $\operatorname{Max} *$ Power, which is not entirely correct. The correct statement, as we will shortly demonstrate, is that inclusion of Max sometimes drives down the statistical significance of Max*Power, and does so for both Max and Max*Power. As we will show in the next section, the reason for this pattern relates to the high multicollinearity between $\operatorname{Max}$ and $M a x *$ Power. While asserting multicollinearity between these variables is the reason for this pattern does not resolve which variable is more important, establishing this phenomenon is at play is a reasonable starting point. We will then turn to tests that will allow us to make inferences about which of the two variables is more important while accounting for this statistical problem.

\section{Including Max and Max * Power in the Estimation}

As we have argued, there is little theoretical justification from the theory in MNS for including Max and Max* Power in the wage equation. ${ }^{4}$ In this section we show that there is a high multi collinearity between Max and $\operatorname{Max} *$ Power and argue that this statistical issue makes it difficult to identify rigging and its drivers with both these variables in the estimation. We think that proper identification of drivers of rigging is better left to tests that exploit cross sectional variation - many of which we had explored in some detail before the paper was published, but were not included in the published version (discussed in Section 4).

\subsection{Multicollinearity with both $\operatorname{Max}$ and $\operatorname{Max} *$ Power in Estimation}

In this section, we discuss the mathematical foundations of estimating variables with high correlations. As a preamble, we start by measuring the

4 Despite the lack of theoretical justification, some earlier drafts of our original paper included both Max and Max* Power in many specifications. These specifications did not survive the refereeing process. Appendix A describes these tables, and the results are provided in Appendix Table 1. The coefficient on the interaction term survives for many measures of power variables even when we include Max. Notably, there is no specification where we find Max is significant and positive while $M a x *$ Power is not. We relegated this comment to a footnote because, given the lack of significance on Max and Max*Power for some variables due to high multi collinearity (over 80\%), these estimations may not entirely convince readers that rigging is being driven by CEOs with power across all measures. This is a task that our new cross sectional tests are better equipped to demonstrate. 
Panel A: Raw Correlations

\begin{tabular}{cccc}
\hline & Max*PowerIndex & Max $*$ Insider $\%$ & Max $* \%$ Appointed \\
\hline $\operatorname{Max}$ & $92 \%$ & $76 \%$ & $77 \%$ \\
\hline
\end{tabular}

Panel B: Within firm Correlations

\begin{tabular}{|c|c|c|c|c|c|c|}
\hline \multicolumn{3}{|c|}{ Max $*$ PowerIndex } & \multicolumn{2}{|c|}{ Max $*$ Insider $\%$} & \multicolumn{2}{|c|}{ Max $* \%$ Appointed } \\
\hline Max & $92 \%$ & & 73 & & $76 \%$ & \\
\hline \multicolumn{7}{|c|}{ Panel C: Variance Inflation Factors (VIFs) } \\
\hline Max & 15.47 & & $\operatorname{Max}$ & 9.03 & $\operatorname{Max}$ & 9.56 \\
\hline Max $*$ Power & 10.08 & Max* & Insider\% & 2.81 & Max*Appointed\% & 3.61 \\
\hline
\end{tabular}

Table 2. Multicollinearity between Max and Max * Power.

Description: In this table we report correlations and multicollinearity statistics between the Max variable and Max interacted with the power measures. Panel A reports raw correlations. Panel B reports the correlations more appropriate for considering the impact of collinearity in a fixed effects specification, namely within firm correlations. Panel C reports Variance Inflation Factors (VIFs) from the MNS regressions, iteratively employing the three power measures - PowerIndex, Insider\% and \%Appointed - including both Max and $M a x *$ Power in the same fixed effects regression.

correlations. Panel A of Table 2 presents the raw correlations between Max and Max* Power for various power variables. These correlations are large for all the power variables. ${ }^{5}$ Since our regressions exploit within firm variation, the more relevant statistic is the within-firm correlation between Max * Power and Max. We report these in Panel B. These correlations are 92\% for Power Index, 76\% for Appointed $\%$ and around $73 \%$ for Insider $\%$. The other standard approach to consider collinearity is to quantify its

5 The fact that the correlation between Max and Max* Power is in excess of $90 \%$ is not surprising when one considers the structure of the Power variable. It has little to do with the nature of the Max variable per se and the correlation is about as large if we replace Max with, say, a variable $x \sim$ $N\left(0, \sigma_{x}^{2}\right)$ that is independent of Power. We will determine the correlation between $x$ and $x *$ Power, using the fact that the Power variable is distributed such that its values of $1,2: \&: 3$ constitute $0.25,0.50: \&: 0.25$ of the sample, respectively. The Covariance between $x$ and $x *$ Power $=\sigma_{x}^{2}[0.25 *$ $1+0.5 * 2+0.25 * 3]=2 \sigma_{x}^{2}$. Hence, the correlation $\rho_{x, x * \text { Power }}=\frac{2 \sigma_{x}^{2}}{\sigma_{x}^{2} \sqrt{1 / 4+2+9 / 4}}=0.94$. 
estimation variance effect using the variance inflation factor (VIF) metric. The VIF conveys how much variance in parameter estimates is induced by multicollinearity. The standard rule is that any VIF over five is problematic, and close to 10 is fatal for inferences. Panel $\mathrm{C}$ of Table 2 shows that when we use PowerIndex as the measure of power, the VIF on both Max and $\operatorname{Max} *$ Power are over 10. For the other power measures, the VIFs on these variables is also quite large. ${ }^{6}$

With these numbers in mind, what happens when correlations among independent variables grow large, approaching unity? Consider a version of the wage equation that we presented earlier, where the firm and time averages have been removed. Let us also focus only on Max and Max * Power, simplified to:

$$
y_{i t}=\beta_{m} \operatorname{Max}_{i t}+\beta_{p} \operatorname{Max}_{i t} * \text { Power }_{i t}+\eta_{i t} .
$$

We know then that:

$$
\begin{aligned}
\widehat{\beta_{m}}= & \frac{\rho_{[y, \text { Max }]}-\rho_{[y, \text { Max } * \text { Power }]} \times \rho_{[\text {Max }, \text { Max } * \text { Power }]}}{\left(1-\rho_{[\text {Max }, \text { Max } * \text { Power }]}\right)^{2}} * \sqrt{\frac{\operatorname{Var}(y)}{\operatorname{Var}(\text { Max })}} \\
\widehat{\beta_{p}=} & \frac{\rho_{[y, \text { Max } * \text { Power }]}-\rho_{[y, \text { Max }]} \times \rho_{[\text {Max }, \text { Max *Power }]}}{\left(1-\rho_{[\text {Max }, \text { Max } * \text { Power }]}\right)^{2}} \\
& * \sqrt{\frac{\operatorname{Var}(y)}{\operatorname{Var}(\text { Max } * \text { Power })}},
\end{aligned}
$$

where $\rho[a, b]$, represents the simple correlation between $a$ and $b$. Under the usual assumptions of $\eta$ being classical, the variance of these parameters, will be:

$$
\begin{aligned}
& \operatorname{Var}\left(\widehat{\beta_{m}}\right)=\frac{\operatorname{Var}(\eta)}{\operatorname{Var}(\operatorname{Max}) \times\left(1-\rho_{[\text {Max }, \text { Max } * \text { Power }]}\right)^{2}} \\
& \operatorname{Var}\left(\widehat{\beta_{p}}\right)=\frac{\operatorname{Var}(\eta)}{\operatorname{Var}(\text { Max } * \text { Power }) \times\left(1-\rho_{[\text {Max }, \text { Max } * \text { Power }]}\right)^{2}}
\end{aligned}
$$

6 Another test to demonstrate multicollinearity is to show that while individually the variables might be insignificant, jointly the same variables explain reasonable variation and are significant. As noted earlier, when both Max and Max*Power are included, they both become individually insignificant when PowerIndex and Appointed\% are used as power measures. While individually insignificant, the joint F-statistic on the two variables, Max and $\operatorname{Max} *$ Power for the specifications that use these variables is significant at least at the $5 \%$ level. 
The implication for the case when $\rho_{[\text {Max,Max*Power }]} \rightarrow 1$ is straightforward; the variance (and thus the standard error) on estimated parameters increases significantly. In fact, $\operatorname{Var}\left(\widehat{\alpha_{1}}\right) \rightarrow \infty$ as $\rho_{[\text {Max,Max*Power }]} \rightarrow 1$. As a result, statistical significance of estimates falls dramatically. This is true both for the estimate on Max and Max*Power. That is what we see in the regressions (Wan's Table 3$).^{7}$

The simple math does not establish our claim that it is powerful CEOs that rig. However, it establishes the fact that, given the high correlation between the two, one cannot identify meaningful estimates and standard errors with both Max and Max * Power in the estimation.

\begin{tabular}{llccc}
\hline & & \multicolumn{3}{c}{ Wan's Standardization } \\
\cline { 3 - 5 } & & Not Switch & Switch & Total \\
\hline \multirow{2}{*}{ Not Switch } & 1,445 & 701 & 2,146 \\
& & $15.8 \%$ & $7.7 \%$ & $23.4 \%$ \\
& \multirow{2}{*}{ Switch } & 4,052 & 2,961 & 7,013 \\
& \multirow{2}{*}{ Total } & $44.2 \%$ & $32.3 \%$ & $76.6 \%$ \\
& & 5,497 & 3,662 & 9,159 \\
& & $60.0 \%$ & $40.0 \%$ & $100.0 \%$ \\
\hline
\end{tabular}

Table 3. Reconciling MNS with Wan: Computing Max.

Description: In this table we report observations that represent a switch in a given performance measure being the better performing measure when compared to the better performing measure in the prior firm-year observation. The matrix outlines how Wan's standardization compares with our standardization.

Interpretation: Wan's standardized performance measures are different than ours. His standardization results in accounting returns being 14 percentage points higher than stock returns. Consequently, as the table shows, Wan's Max variable - which captures the better performing measure in a period - misses over 4,000 of our switches, which is about $44 \%$ of our entire sample. Thus, in Wan's estimation, switching between measures, necessary for identification, is infrequent and outlierbased.

7 The impact on coefficients themselves is a bit ambiguous, and can depend on the data. This is because as $\rho_{[\text {Max,Max } * \text { Power }]} \rightarrow 1$, it must be the case that $\rho_{[y, \text { Max } * \text { Power }]} \approx \rho_{[y, \text { Max }]}$. Therefore, as $\rho_{[\text {Max }, \text { Max } * \text { Power }]} \rightarrow 1$, the estimates $\beta_{m}$ and $\beta_{p}$ become indeterminate, since both the numerator and denominator tend to zero (that is, estimates become $=\frac{0}{0}$ ). 


\section{Reconciling Data and Results: Max Computation}

Before we can present tests that exploit cross sectional variation to pinpoint the driver of rigging, we want to discuss the fact that our results differ from Wan, even when he is replicating our baseline specification (or we, his). We begin with Equation (1) augmented with Max. To illustrate the differences, we focus on the Insider\% measure of power, in the following equation:

$$
Y_{i t}=\left\{\begin{array}{c}
\beta_{1} \text { Insider }_{i t}+\beta_{2} z \text { RStock } \\
\\
\quad+\beta_{4} \operatorname{Max}_{i t}+\beta_{3} z \text { ROA } \text { Max }_{i t} * \text { Insider }_{i t}+\gamma X_{i t}+\mu_{i}+\delta_{t}+\eta_{i t}
\end{array}\right\} .
$$

In his main replication results (column 8 of Table 3 ) Wan finds that $\beta_{4}=$ 0.055 and $\beta_{P}=0.062$. Neither is significant. This is in stark contrast to what we find. In a similar specification reported in Appendix Table 1, column 5, we find that $\beta_{4}=0.028$ and is insignificant, but $\beta_{P}=0.159$ and is significant at the $5 \%$ level. ${ }^{8}$

The CFR journal editor facilitated our trading of data with Wan to uncover the discrepancy. In doing the reconciliation, we find that if we re-estimate this specification in Wan's data we obtain estimates that are very similar to Wan's estimates for all of his variables except for the standardized performance measures and Max, where we find an insignificant $\beta_{4}=0.049$ and a statistically significant $\beta_{P}=0.124$. Thus, the discrepancy stems from differences in standardizing performance measures. This makes sense since Wan's power measures come directly from our website, so there are only minor sample differences there.

In MNS we were testing the wage equation implied by our theory, which includes a variable that captures the return of the ex-post performance measure that does relatively better that period for firms with powerful CEOs (Max* Power). The setting was one where boards pay CEOs based on performance which is captured by two measures, one being a stock return measure and the other being accounting performance. However, since accounting and stock returns have different distributions in the data, one cannot cardinally compare returns of the two to say which measure does better when we construct Max. Therefore, we proposed in MNS to standardize these measures to make the accounting and stock returns comparable, in

8 Note that these differences are also clear when we compare the baseline results without including the Max term with results reported in Wan's paper. 
order to be able to pick which of the measures perform more favorably in a period. Accordingly, we had standardized the measures as follows:

$$
\begin{aligned}
z R O A_{i t} & =\frac{R O A_{i t}-\overline{R O A}_{t}}{\sigma_{t}^{\overline{R O A}}} \\
z R S t o c k_{i t} & =\frac{\text { Rstock }_{i t}-\overline{\text { RStock }}_{t}}{\sigma_{t}^{\overline{\text { Stock }}}} .
\end{aligned}
$$

$\left\{\overline{R O A_{t}}, \overline{\text { RStock }_{t}}\right\}$ and $\left\{\sigma_{t}^{\overline{R O A}}, \sigma_{t}^{\overline{R S t o c k}}\right\}$ are firm i's industry mean return and standard deviation, respectively, for accounting (ROA) and stock (RStock) in year $t$.

Wan follows MNS in making accounting and stock returns comparable, but he standardizes using a larger set of firms, many of which are not part of his subsequent estimation. When doing his standardization, Wan finds the means and standard deviations for the standardized variables are $(0.070,0.80)$ for $z R O A$ and $(-0.074,0.752)$ for $z R S t o c k$. Thus, a 14 returns percentage point differential results from Wan's construction. This is a large number!. When the maximum is taken over these measures, accounting returns is the better performing measure in almost two-thirds of the cases. This differs from our sample since our better performing measure picks up accounting returns in about half of the observations.

The first implication from Wan's construction is that his Max only picks up stock returns as the better measure in cases with either extremely good stock performance or extremely poor accounting returns. This fact is going to be important to understanding why Wan gets different results than we do when he asserts that rigging only occurs in firms with low stock performance.

The second implication concerns identification. Because the estimating equations include the levels of performance measures as well as the Max taken over them and firm fixed effects, the only identification of the coefficient on Max comes from firms which sometimes have accounting returns being the better measure and sometimes have stock returns as the Max. Wan's construction eliminates half of such switchers because his measure systematically picks accounting returns as the better performing measure.

To illustrate this clearly, in Table 3 we present observations which represent a switch in a given performance measure being the better performing measure when compared to the better performing measure in the prior firmyear observation. As is evident, Wan's Max variable misses over 4,000 of 
our switches, which is about $44 \%$ of our entire sample. Given this large difference, it is not surprising that we identify effects with a Max $*$ Insider $\%$ variable in the presence of Max while he is unable to do so. In other words, Wan does not exploit the same variation in the data even when he replicates our baseline results.

The third implication that results from Wan's construction of Max concerns multicollinearity. In particular, as we discussed in the prior section, multicollinearity renders inference ineffective when Max and Max*Power are included together. The fact that Wan's method also makes Max more likely to systematically pick accounting returns potentially accentuates these concerns in that Max (and thus $\operatorname{Max} *$ Power) also becomes increasingly collinear with $z R O A .^{9}$

Finally, Wan writes that our results do not hold in the absence of winsorizing. In fact, our results are quite robust to not winsorizing, which we show in Table 4. In some sense, it is not clear why we are writing this paragraph since Wan seems to show that our results are robust to not winsorizing, for which we thank him. Even when he uses his own measure of Max, the only measure of power where Wan finds that the coefficient drops in size and becomes insignificant relates to insider percentage measure of power (Wan, Table 8). These results are reproduced in the first six columns of Table 4. As we have discussed in detail, this is purely an artifact of the way Wan constructs Max. In short, the only observations which identify his coefficients on Max and Max*Power - based on his measure of Max - are extremely high stock returns and extremely low accounting returns. Thus, by construction, Wan finds weaker results when he does not winsorize. To illustrate this, we repeat estimation of Wan's specification with insider percentage measure of power, using our measure of Max and with no winsorization. As is shown in column 7 , the coefficient estimate is still extremely large and significant at the $1 \%$ confidence level.

9 It is also unclear what inferences can be derived from standardizing performance measures using firms that are omitted from compensation analysis. For instance, if Wan largely omits small firms in compensation analysis, one could argue that it is also reasonable to exclude such firms when standardizing performance measures. The reason is that such firms are less likely to be in the comparison set of boards of larger firms when they make compensation decisions. Alternatively, if Wan omits firms that are larger in compensation analysis, he could be introducing a bias in his estimates by only including these firms for standardizing performance measures. Regardless, this choice by Wan is non-trivial and creates differences between results in Wan and MNS even for the simple baseline specification. 


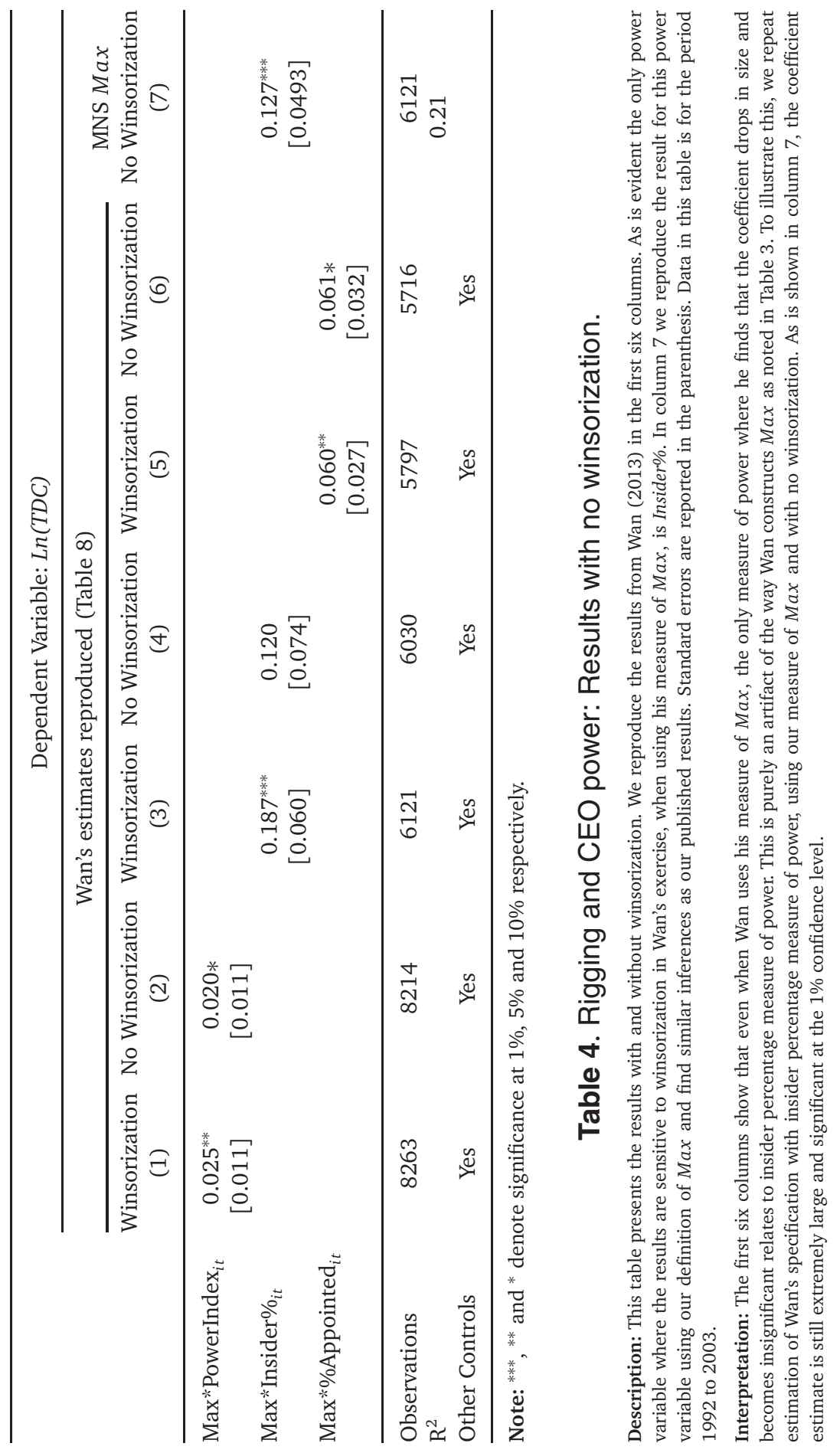


Overall, given the fundamental differences in how the main variable Max has been constructed, we think that there is sufficient reason to doubt the nature of replication tests presented by Wan, and the criticism therein.

\section{Cross Sectional Evidence on Power in Rigging}

Irrespective of the issues that we believe are questionable in Wan's replication tests, we now move towards presenting tests that exploit cross sectional variation to assess whether rigging is identified only off firms with powerful CEOs. It is worth mentioning that, before we published our original paper, we had estimated specifications including both Max and Max * Power in the CEO wage equation. These tests appeared in early drafts of our work where we had used slightly different power measures, which evolved with comments of the referees (see Appendix Table 1 and Appendix Table 2). However, we wanted to note that these results had convinced us that rigging is largely driven by powerful CEOs before we wrote the first draft of the paper. We now take one step further and exploit the cross section to show again that rigging only happens in firm-years with powerful CEOs.

We present these additional results in two subsections. The idea of the first subsection is to estimate a specification that looks very much like our main estimating equation, but we break down the sensitivity of compensation to Max by levels of power. This specification has the advantage of illustrating clearly that these sensitivities are not the same across firms with different CEO power as Wan predicts, but rather rigging occurs among firms with powerful CEOs.

The second subsection adds two other cross sectional specifications. First, we collapse the time series data for each firm to obtain a single cross section in the spirit of Bertrand et al. (2004) and look at the splits of the data by power. Second, we estimate a between-effects regression following Wooldridge (2002) and Cameron and Trivdei (2005). In both these tests we find the same result: rigging occurs only by powerful CEOs.

\subsection{Breaking Out the Interaction}

A straightforward way to assess which part of the sample drives the results is

to allow the coefficient on Max to vary in the cross section according to CEO power. Doing so results in variables that are only moderately correlated, but 
can capture both the level effect of Max and the interaction Max * Power. More specifically, the baseline fixed effects specification we consider is:

$$
\begin{aligned}
y_{i t}= & \beta_{p_{H}} I_{\text {Power }_{i t}=\text { High }} * \text { Max }+\beta_{p_{M}} I_{\text {Power }_{i t}=\text { Medium }} * \text { Max } \\
& +\beta_{p_{L}} I_{\text {Power }_{i t}=\text { Low }} * \text { Max }+\eta_{i t},
\end{aligned}
$$

where, $I_{\text {Power }_{i t}=\text { High, Medium or Low }}$ are dummy variables that indicate whether the CEO power in the firm is high, medium or low, taking tertiles of the cross section by year. This equation maintains the baseline specification in MNS, while the variables $I_{\text {Power }_{i t}}=$ High $_{\text {ig }} *$ Max,$I_{\text {Power }_{i t}}=$ Medium $*$ Max and $I_{\text {Power }_{i t}}=$ Low $*$ Max have only moderate correlation. For brevity, in this equation we have suppressed other control variables and firm and time fixed effects, which are also employed when estimating this specification. Wan's argument, if correct, would imply that the estimated effect on Max is significant across the three groups of firms.

Table 5 reports the results from this analysis for each of the three measures of power. The rigging coefficient for the most powerful CEOs is large and statistically significant. In addition, the coefficient on rigging is largely absent for CEOs who have medium and low power. This is the case for all three measures of CEO power. These results support the claims in MNS.

\subsection{Splits and Between Estimator}

We now turn to more tests, with the goal of leaving no doubt that the ability to rig requires $\mathrm{CEO}$ power. In the prior table, we looked at compensation sensitivity to Max for different levels of power in a firm fixed effects ('withinfirm') setting. By contrast, this section uses the structure which is broadly in the spirit of a 'between' estimator to isolate sensitivity of compensation to Max in the firm cross section. The idea is in the spirit of Bertrand et al. (2004), who suggest that panel estimates should be robust to collapsing to time series averages across firms. Our estimation specification and notation follow Wooldridge (2002) and Cameron and Trivdei (2005).

We start by classifying and sorting firms into three levels of power (using a blend of power variables). We superscript these three power levels as $p \subseteq\{1$ : low, 2 : medium, $3:$ high $\}$. We apply two approaches to assign the firms into these power levels. The two methods use all of our power measures to form a combined continuous variable, which allows for evenly distributed low, medium, and high power sample splits. First, we make an 
Panel A: Differential Effects using same Baseline specification

\begin{tabular}{|c|c|c|c|c|c|c|}
\hline & \multicolumn{6}{|c|}{ Dependent Variable: $\operatorname{Ln}(T D C)$} \\
\hline & (1) & (2) & (3) & (4) & (5) & (6) \\
\hline \multirow{2}{*}{$\begin{array}{l}\text { Max } * \text { High } \\
\text { Power }\end{array}$} & $0.0838^{* * *}$ & $0.0762^{* *}$ & & & & \\
\hline & {$[0.0310]$} & {$[0.0308]$} & & & & \\
\hline \multirow[t]{2}{*}{ Max $*$ Medium } & $0.0530 *$ & $0.0464 *$ & & & & \\
\hline & {$[0.0319]$} & {$[0.0278]$} & & & & \\
\hline \multirow{2}{*}{$\begin{array}{l}\text { Max } * \text { Low } \\
\text { Power }\end{array}$} & 0.0453 & 0.0376 & & & & \\
\hline & {$[0.0349]$} & {$[0.0307]$} & & & & \\
\hline \multirow{2}{*}{$\begin{array}{l}\text { Max } * \text { High } \\
\text { Insider }\end{array}$} & & & $0.104^{* * *}$ & $0.106^{* * *}$ & & \\
\hline & & & {$[0.0333]$} & {$[0.0374]$} & & \\
\hline \multirow{2}{*}{$\begin{array}{l}\text { Max } * \text { Medium } \\
\text { Insider }\end{array}$} & & & 0.0342 & 0.0393 & & \\
\hline & & & {$[0.0368]$} & {$[0.0349]$} & & \\
\hline $\operatorname{Max} *$ Low & & & $0.0625 *$ & $0.0618 *$ & & \\
\hline Insider & & & {$[0.0339]$} & {$[0.0352]$} & & \\
\hline $\operatorname{Max} *$ High & & & & & $0.0914^{* * *}$ & $0.0858^{* *}$ \\
\hline \multirow{2}{*}{ \%Appointed } & & & & & {$[0.0315]$} & {$[0.0366]$} \\
\hline & & & & & 0.0496 & 0.0564 \\
\hline $\begin{array}{r}\text { Max } * \text { Medium } \\
\% \text { Appointed }\end{array}$ & & & & & {$[0.0389]$} & {$[0.0387]$} \\
\hline $\operatorname{Max} *$ Low & & & & & 0.0431 & 0.0348 \\
\hline \%Appointed & & & & & {$[0.0416]$} & {$[0.0377]$} \\
\hline Observations & 8263 & 8190 & 6121 & 5834 & 5797 & 5474 \\
\hline $\mathrm{R}^{2}$ & 0.239 & & 0.219 & & 0.222 & \\
\hline Time \& Firm F.E. & Yes & Yes & Yes & Yes & Yes & Yes \\
\hline Other Controls & Yes & Yes & Yes & Yes & Yes & Yes \\
\hline
\end{tabular}

Note: ${ }^{* * *},{ }^{* *}$ and ${ }^{*}$ denote significance at $1 \%, 5 \%$ and $10 \%$ respectively.

Table 5. Is the effect of max equivalent across power levels? Breaking out the interaction by power tertiles.

Description: The dependent variable is the natural log of the total compensation of the CEO. We present the specifications interacting Max with dummy variables that capture whether the firm has CEO power in one of the terciles (High, Medium or Low). We iteratively employ the three power measures - PowerIndex, Insider\% and \%Appointed. Controls employed are same as in MNS, except that power measures enter as dummy variables indicating the tertile of power level. Odd columns are estimated with firm fixed effects and lagged performance included, and even columns are GLS-AR1 specification as in MNS. Standard errors, which are robust in the fixed effects columns, are reported in the parenthesis. Data are for the period 1992 to 2003.

Interpretation: For all three measures of CEO power, the rigging coefficient for the most powerful CEOs is large and statistically significant, while it is largely absent for CEOs who have medium and low power. These results support the claims in MNS that it is powerful CEOs that induce boards to shift the weight on performance measures towards measures that are ex-post more favorable. 
equally-weighted index across the three measures. Since the three measures differ on the scale, we standardize Insider\% and \%Appointed to have the mean (2) and standard deviation (0.75) of PowerIndex. We then agnostically add the three measures to create a continuous variable index and take tertile splits. The second approach is to take the first principal component annually in the cross section, and then split the score variable into tertiles.

In all the tests presented in this section, our goal is to collapse compensation and covariates into one observation per firm. We subtract out firm averages for all the variables to remove unobserved heterogeneity that is always a feature of compensation. ${ }^{10}$ We denote demeaned compensation as: $\tilde{y}_{i t}=y_{i t}-\bar{y}_{i}$. and demeaned covariates as $\widetilde{X}_{i t}=X_{i t}-\bar{X}_{i \text {. }}$, where index $i$ refers to the firm and index $t$, time.

Before getting to our main, collapsed specification, in Panel A of Table 6, we begin with the simple exploration of pooled regressions with the demeaned variables, split by power level. We regress $\tilde{y}_{i t}$ on $\tilde{X}_{i t}$, without including any power terms but estimated within sample-splits that are based on power mixture in columns $1-3$ and based on principal component of power variables in columns 4-6. This specification differs only marginally from Table 5 but is useful as a starting point for the analysis that follows. Not surprisingly, as before, we find that rigging only happens in high power CEO firms.

We now turn to the main test where we derive the between estimator by collapsing the data. If we write out the compensation function of this specification while allowing for the possibility of unobserved firm heterogeneity specific to a power level $\left(c_{i}^{p}\right)$, we would have:

$$
\tilde{y}_{i t}=\beta_{0}^{p}+\tilde{X}_{i t}^{\prime} \beta_{1}^{p}+c_{i}^{p}+\varepsilon_{i t}, \text { for all } i \text { in } p .
$$

Here, firm is denoted by $i$ and power level is denoted by $p$. It is this unobserved firm heterogeneity, $c_{i}^{p}$, using Wooldridge's (2002) notion, that motivates the use of panel models, like the between or within models we are using here. Although we have manually taken out a fixed effect, a concern is that something specific to the firm, when it is at a high, medium or low power level, is unobserved and correlated with one of the $X^{\prime}$ s. Thus, we employ the

10 Note that, rather than trying to explain overall levels of compensation, our modest goal is to focus on the sensitivity of pay to Max. Specifically, we do this de-meaning prior to running an estimation so that variables are demeaned by their firm average across the entire sample, rather than demeaning based on the sample of firms sorted into a tertile. 
Panel A: Pooled Estimator on Aggregated Power Index

\begin{tabular}{|c|c|c|c|c|c|c|}
\hline & \multicolumn{6}{|c|}{ Dependent Variable: $\operatorname{Ln}(T D C)$} \\
\hline & \multicolumn{3}{|c|}{ Power Mixture } & \multicolumn{3}{|c|}{ Principal Component } \\
\hline & $\begin{array}{l}\text { Low } \\
(1)\end{array}$ & $\begin{array}{l}\text { Medium } \\
\text { (2) }\end{array}$ & $\begin{array}{l}\text { High } \\
\text { (3) }\end{array}$ & $\begin{array}{l}\text { Low } \\
(4)\end{array}$ & $\begin{array}{c}\text { Medium } \\
\text { (5) }\end{array}$ & $\begin{array}{l}\text { High } \\
(6)\end{array}$ \\
\hline Max & $\begin{array}{c}-0.0843 \\
{[0.0669]}\end{array}$ & $\begin{array}{c}0.0681 \\
{[0.0467]}\end{array}$ & $\begin{array}{r}0.211^{* * *} \\
{[0.0463]}\end{array}$ & $\begin{array}{c}-0.0269 \\
{[0.0631]}\end{array}$ & $\begin{array}{c}0.0727 \\
{[0.0547]}\end{array}$ & $\begin{array}{c}0.156^{* * *} \\
{[0.0411]}\end{array}$ \\
\hline Observations & 1836 & 1861 & 1756 & 1806 & 1871 & 1776 \\
\hline $\mathrm{R}^{2}$ & 0.13 & 0.299 & 0.249 & 0.145 & 0.245 & 0.318 \\
\hline Other Controls & Yes & Yes & Yes & Yes & Yes & Yes \\
\hline
\end{tabular}

Panel B: Between Estimator on Aggregated Power Index

\begin{tabular}{|c|c|c|c|c|c|c|}
\hline & \multicolumn{6}{|c|}{ Dependent Variable: $\operatorname{Ln}(T D C)$} \\
\hline & \multicolumn{3}{|c|}{ Power Mixture } & \multicolumn{3}{|c|}{ Principal Component } \\
\hline & $\begin{array}{l}\text { Low } \\
(1)\end{array}$ & $\begin{array}{l}\text { Medium } \\
\text { (2) }\end{array}$ & $\begin{array}{l}\text { High } \\
\text { (3) }\end{array}$ & $\begin{array}{c}\text { Low } \\
(4)\end{array}$ & $\begin{array}{l}\text { Medium } \\
\text { (5) }\end{array}$ & $\begin{array}{l}\text { High } \\
\text { (6) }\end{array}$ \\
\hline Max & $\begin{array}{c}-0.338^{* * *} \\
{[0.128]}\end{array}$ & $\begin{array}{c}0.143 \\
{[0.108]}\end{array}$ & $\begin{array}{c}0.443^{* * *} \\
{[0.115]}\end{array}$ & $\begin{array}{r}-0.0755 \\
{[0.104]}\end{array}$ & $\begin{array}{c}0.184 \\
{[0.114]}\end{array}$ & $\begin{array}{c}0.281^{* * *} \\
{[0.0830]}\end{array}$ \\
\hline Observations & 1836 & 1861 & 1756 & 1806 & 1871 & 1776 \\
\hline $\mathrm{R}^{2}$ & 0.192 & 0.269 & 0.224 & 0.247 & 0.252 & 0.391 \\
\hline Other Controls & Yes & Yes & Yes & Yes & Yes & Yes \\
\hline
\end{tabular}

Note: ${ }^{* * *},{ }^{* *}$ and ${ }^{*}$ denote significance at $1 \%, 5 \%$ and $10 \%$ respectively.

Table 6. Rigging and CEO power: Cross sectional evidence.

Description: This table presents split estimations providing cross sectional evidence using a pooled and between estimator. We begin by creating three splits of the data based on tertiles of the mixture of power variables (described in the text) and tertiles of the first principal components of the power variables. We then take out the firm fixed effects of log consumption and all the covariates by subtracting out the firm mean across the sample period. As described in the text, we do this de-meaning prior to running an estimation so that variables are demeaned by their average across the entire firm sample, rather than demeaning based on the sample appearing in the power split. Panel A presents a pooled estimation of rigging, where we simply regress (everything de-meaned) compensation on Max and other controls in MNS specifications in tertiles created based on power mixture and power based principal component. Panel B presents between effects model estimates within the same sorts. Standard errors are reported in the parenthesis. Data in this table is for the period 1992 to 2003.

Interpretation: The magnitude and significance of the Max coefficients indicate the sensitivity of average compensation to average rigging across the low, medium, and high sub-samples of CEO power. These results support the claims in MNS that it is powerful CEOs that induce boards to shift the weight on performance measures towards measures that are ex-post more favorable. 
between estimator to collapse and identify off the cross section. The between effects equation is:

$$
\overline{\widetilde{y}}_{i}=\beta_{0}^{p}+\overline{\widetilde{X}}_{i}^{\prime} \beta_{1}^{p}+\left(c_{i}^{p}-\beta_{0}^{p}+\bar{\varepsilon}_{i}\right), \text { for all } i \text { in } p
$$

The bars over the variable indicate the taking of a time-series average of the variable in the firm while it is at power level $p$. Note that, though we demeaned variables at the firm level, the dependent variable when collapsed within each power tertile is not mechanically zero. The reason is that, with the power subsampling, firms can switch across power tertiles over time. It is these switching firms that serve to identify this equation. The important assumption of the between estimator is that the composite error term $\left(c_{i}^{p}-\right.$

$\beta_{0}^{p}+\bar{\varepsilon}_{i}^{p}$ ) is independent of the regressors $\bar{X}_{i}^{p^{\prime}}$, once the firm averaging has occurred.

Table 6, panel B presents the between effects estimate equivalent of Panel A. The pattern is the same in this rather stringent estimation. Rigging can only be detected significantly when CEOs have power.

In Appendix B, we present a modified between effects model for more robustness. The idea therein is to use the between effects model and assumptions for identification, but allow the performance measures to vary over time. Since, in essence this formulation is a combination of the pooled and between estimator, it is not terribly surprising that our results remain robust (see Appendix Table 3). Nevertheless, we present it because the formulation leads to a more natural interpretation of the rigging coefficients.

Math aside, we want to conclude by making sure that we convey the intuition of what we have shown. We take time-series averages of firm compensation within tertiles formed on the basis of CEO power. We then asked, "Does sensitivity of average compensation to average rigging vary across the low, medium, and high samples of power?" Table 6 shows that the magnitudes and significance of the Max coefficients across power subsamples, strongly support our view of rigging with cross sectional analysis.

\section{Is Rigging General? Analyzing Settings}

Having addressed Wan's concern about the role of power in CEO compensation sensitivity to Max, we move to his other main criticisms. His arguments build a view that rigging is not a general concept, but rather an empirical 
artifact that holds only in certain settings. In particular, Wan argues that the rigging results from optimal repricing of options after low stock performance and new, externally-hired CEOs. In this section, we discuss these settings as well as his findings on rigging for non-CEO compensation.

\subsection{Is Rigging Just an Artifact of Optimal Repricing of Options?}

In his Table 7, Wan (2013) introduces the variables Max *lowstock and Max *lowroa in addition to the Max variable. Here lowstock and lowroa are indicators of whether the firm's stock returns and accounting returns are below the median of the Standard \& Poor's (S\&P) 1500 firms in ExecuComp. His models 1-3 indicate that Max interacted with low stock returns is significant, while Max is no longer significant. Wan argues that this suggests that the findings on rigging may be driven by (optimal) repricing of options. ${ }^{11}$

Our first reaction was that it would have been nice if Wan had produced direct empirical evidence that links Max * lowroa and Max *lowstock to optimal repricing of options. Given the lack of direct evidence, it was unclear to us why these results suggested optimal repricing of options rather than just providing additional evidence that hones in on rigging. As observed earlier, the loss in value and performance associated with the shift in loading on performance measures we document is not supportive of optimal re-contracting.

However, this point is perhaps moot, in that when we re-ran the tests using Wan's calculation of Max and our calculation of Max, we find very different results. Our Table 7 reports this reconciliation. For brevity, we only present results with power index as a measure of power. Column 1 of Table 7 reproduces Wan's Table 7, column 1. In our replication we find results consistent with Wan (unreported). In column 2, however, we use all of Wan's variables except we use our measure of Max instead of his measure of Max as a level and in the interactions. We now find that Wan's assertion that "rigging occurs when firms suffer from poor stock performance and does not occur when firms suffer from poor accounting performance" does not hold. In particular, we find that Max *lowroa is in fact significant in column 2.

11 As already mentioned earlier, we do not understand the economic rationale or underlying model that Wan has in his mind to expect compensation of all CEOs to be related to Max in equilibrium. In our model, sensitivity of CEO compensation to Max is driven by firms with powerful CEOs. Therefore, in our opinion, Wan's emphasis on the form Max in his specifications and interpreting this sensitivity with low and high returns is even more unclear. 


\begin{tabular}{|c|c|c|c|}
\hline & \multicolumn{3}{|c|}{ Dependent Variable: $\operatorname{Ln}(T D C)$} \\
\hline & (1) & (2) & (3) \\
\hline $\operatorname{Max}^{\text {wan }}$ & $\begin{array}{c}0.014 \\
{[0.034]}\end{array}$ & & \\
\hline Max $^{\text {wan }} *$ Lowrstock & $\begin{array}{c}0.055^{* *} \\
{[0.028]}\end{array}$ & & \\
\hline $\operatorname{Max}^{\text {wan }} *$ Lowroa & $\begin{array}{c}0.009 \\
{[0.033]}\end{array}$ & & \\
\hline Max & & $\begin{array}{c}0.0435 \\
{[0.0384]}\end{array}$ & \\
\hline Max $*$ Lowrstock & & $\begin{array}{r}-0.00116 \\
{[0.0344]}\end{array}$ & \\
\hline Max $*$ Lowroa & & $\begin{array}{r}0.0539 * \\
{[0.0300]}\end{array}$ & \\
\hline Max $*$ PowerIndex & & & $\begin{array}{r}0.0218 * \\
{[0.0127]}\end{array}$ \\
\hline Max $*$ PowerIndex $*$ Lowrstock & & & $\begin{array}{c}-0.0042 \\
{[0.0141]}\end{array}$ \\
\hline Max $*$ PowerIndex $*$ Lowroa & & & $\begin{array}{r}0.0245 * \\
{[0.0131]}\end{array}$ \\
\hline Lowrstock & $\begin{array}{c}-0.0600^{* * *} \\
{[0.0209]}\end{array}$ & $\begin{array}{r}-0.0316 * \\
{[0.0184]}\end{array}$ & $\begin{array}{c}-0.0299 \\
{[0.0195]}\end{array}$ \\
\hline Lowroa & $\begin{array}{c}-0.110^{* * * *} \\
{[0.0418]}\end{array}$ & $\begin{array}{r}-0.111^{* * *} \\
{[0.0382]}\end{array}$ & $\begin{array}{r}-0.113^{* * * *} \\
{[0.0383]}\end{array}$ \\
\hline Observations & 7597 & 7577 & 7577 \\
\hline R-squared & 0.41 & 0.233 & 0.233 \\
\hline Time \& Firm F.E. & Yes & Yes & Yes \\
\hline Other Controls & Yes & Yes & Yes \\
\hline
\end{tabular}

Note: ${ }^{* * *},{ }^{* *}$ and ${ }^{*}$ denote significance at $1 \%, 5 \%$ and $10 \%$ respectively.

Table 7. Under what performance settings does rigging occur? Robustness to repricing of options setting.

Description: The dependent variable is the natural $\log$ of the total compensationof the CEO. In column 1, we reproduce Wan's (2013) result using his definition of Max. In column 2 and 3, we use definition of Max as in MNS. We estimate the standard fixed effects specification of MNS, with all the controls and performance measures. Robust standard errors are in parentheses. Data are for the period 1992 to 2003.

Interpretation: In column 1 we reproduce Wan's findings using his Max variable, which as noted in Table 3 differs from our variable. Since the vast majority of observations in Wan's construction of Max have accounting returns higher than stock returns, which include all the observations captured by lowstock, this variable turns out to be more important in his analysis. In column 2, we use all of Wan's variables except we use our measure of Max instead of his measure of Max. Wan's assertion that rigging occurs when firms suffer from poor stock performance and does not occur when firms suffer from poor accounting performance does not hold. Column 3 reports estimates from the same setup, using Wan's measures of low performance and other variables, except that it uses our measure of Max*Power instead of Max. The coefficient on Max * Power is positive and significant, consistent with MNS. 
The intuition for Wan's results comes directly from his standardization. Since the vast majority of observations in his construction of Max have accounting returns higher than stock returns, which include all the observations captured by lowstock, it is not terribly surprising that this variable turns out to be more important when he does his analysis. ${ }^{12}$

We want to take this result one step further. In this section, we have naively only used Max in the specification as Wan prefers. We now assess if the setting being used by Wan provides evidence consistent with our earlier results. Column 3 reports estimates from the same setup, using Wan's measures of low performance and other variables, except that it uses our measure of Max * Power instead of Max. The coefficient on Max * Power is positive and significant, as usual, consistent with MNS.

It is also worth noting that a previous (working paper) version of MNS had examined a similar issue. In the appendix, we reproduce the table from our 2007 version of the paper that we had submitted to JF, which demonstrates that the shift towards the measures that are ex-post more favorable occurs for both stock and accounting returns (Appendix Table 2).

We conclude that our original interpretation of rigging is robust to concern that we are just potentially picking up stock repricing in low stock performance observations. Thus, overall, we feel confident that the optimal repricing of stock options story - especially for all the firms in ExecuComp - is not likely to be driving our findings.

\subsection{Is Rigging Done Only by Newly Hired External CEOs?}

Wan's other story is that rigging is an artifact of the manner in which new CEOs are hired and compensated. He presents results that seem to show that rigging is confined to new CEO hires. This is taken to be evidence against rigging by powerful CEOs since, as he contends, new CEOs lack power.

The interpretation of these findings, even if true, is again unclear. The assumption that newly hired CEOs lack power is a supposition. While some CEOs may gain more power as their tenure increases, a newly arriving CEO

12 In the data lowstock is strongly correlated with the cases in which $z R O A>z R S t o c k$, and lowroa is strongly correlated with the cases in which $z R O S>z R O A$. In such a scenario, $M a x *$ lowstock could be represented as $z R O A * I_{z R O A>z R S t o c k}$ and $M a x *$ lowroa could be represented as $z R O S * I_{z R S t o c k>z R O A}$. The two variables Max*lowstock and Max*lowroa thus effectively span the space of the Max variable. As pointed before, in our view, the form Max is effectively only parsing out specific instances in which powerful CEOs might be rigging their pay. 
may well have significant power as well. For instance, if the new CEO has been brought in to deal with a firm's poor performance, she may have substantial power if the board is particularly keen on retaining her. Indeed, in the data, new external CEOs have higher compensation. In particular, using Wan's categorization of new external CEO firms, median compensation is \$2.95 million for external hires and \$2.01 million for everyone else.

To investigate Wan's hypothesis directly, we use the definition in Wan of a new externally-hired CEO: namely, a CEO who has been hired in the last year and who has also joined the firm within the last year. ${ }^{13}$ Using ExecuComp variables for tenure and the year the executive joined firm, we set out to replicate Wan's Table 6. Table 8 presents our rigging results for the three power measures with the newly hired CEOs excluded. Columns 1-3 show that our rigging results are quite robust to excluding new external CEOs, and quite different from Wan's results.

Because we fail to find evidence for the argument Wan is making, we next reconcile the reasons behind the discrepancy. For comparison, we start with Wan's data and with all of his variables. Column 4 of Table 8 reproduces Wan's result without filtering out the new external hires. We just focus on one power variable for demonstration. Next, in column 5 of Table 8 we again use Wan's data with all of his variables, this time filtering based on Wan's variable that indicates the external new CEOs. We are able to replicate his result.

We now explain why are our result (column 1 of Table 8) is so different from his. One reason, as we have already noted before, is that Wan's main specification differs greatly from ours because of the construction of his Max variable. This leads to differences in the starting point itself. The other reason could have to do with construction of the external new CEO measure. When we calculate our new external CEO hire measure we find that it has only a 0.45 correlation with Wan's measure of external CEOs. While we calculate new external hires as being CEOs in ExecuComp with company tenure equal to zero and CEO tenure equal to zero, in Wan's codebook, he defines the term as:

Externally-hired new CEO (EXTNEWCEO): The individual is an externally-hired new CEO if he/she (i) is not the founder of the company and (ii) has served the firm for less than one year prior to taking the CEO position in the compensation year.

13 We calculate new external hires as being CEOs in ExecuComp with company tenure equal to zero and CEO tenure equal to zero. 


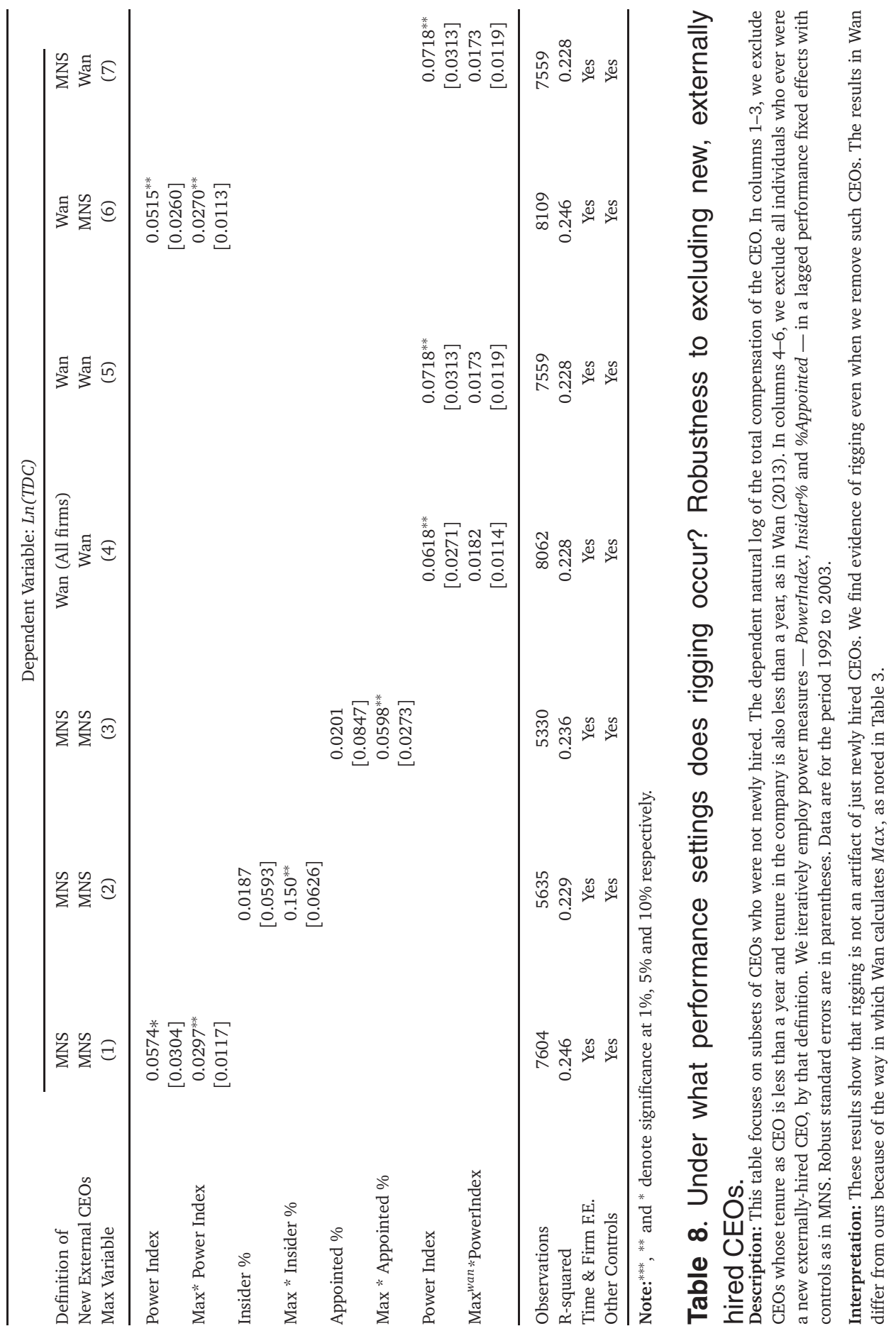


We cannot figure out why these alternative ways of constructing externally-hired CEOs lead to such differences. Regardless, in column 6 of Table 8 we estimate the regression with Wan's definition of new external CEOs and Wan's data but using our definition of Max. The results remain the same as what we reported earlier. More specifically, in column 6, we find that the coefficient on Max * Power is significant, even for observations which are not new external CEOs. For completeness, in column 7 we re-estimate the specification with our definition of new external CEOs but using Wan's definition of Max. We find that the results we had found in column 1 disappear. Thus, we conclude that rigging is not an artifact of just newly hired CEOs. We find evidence of rigging even when we remove such CEOs. The results in Wan differ from ours because of the way in which Wan calculates Max.

Overall, we find that our result that there is rigging in pay by powerful CEOs is present even if we remove externally-hired CEOs. Moreover, even if Wan's findings were true, there is no theoretical basis to argue that newly hired CEOs would not have substantial power when they were hired - and as a result would not be able to engage in rigging their compensation.

\subsection{Other Findings}

\subsubsection{Evidence on Non-CEOs}

Wan also examines the topic of whether powerful CEO rigging effects also impact the total compensation received by non-CEO top executives. His Table 5 follows the specifications of our published paper and report the rather interesting finding that the evidence of rigging seems also to be present in the total compensation of the non-CEO top executives.

Wan, somehow takes this to be evidence against the CEO rigging argument of MNS, though it is not clear why. As we discuss in MNS, the notion of CEO power is about the relative power of the CEO in relation to the board, using indicators such as the formal positions held by the CEO, percentage of insiders on the board and the fraction of the board appointed during the CEO's tenure. Hence, while it is not part of the model and analysis in MNS, it is perfectly plausible that if there are, for instance, more insiders on the board this could benefit the non-CEO top executives as well. It is also plausible that powerful CEOs, seeking to build unity and loyalty in the 
management team, would want the rest of the top managers to be rewarded in ways that are similar to their own incentive pay.

Furthermore, if the situation is one in which the CEO is being compensated based on a particular performance measure - it would be peculiar for the board to use a very different metric for the rest of the senior management. Therefore, it seems reasonable to argue that if the board is compromised and compensates the CEO based on rigged performance indicators, then senior management of the whole firm may benefit as well. ${ }^{14}$ Regardless, by itself, it is unclear why rigged compensation of non-CEO managers contradicts the MNS hypothesis. If anything, this appears to be a useful extension of the ideas in MNS.

In Wan's empirical analysis in Table 5, he again introduces Max as an additional variable and finds, not surprisingly, that the significance of Power * Max is affected. In our Table 9, we show that the evidence Wan presents is stronger in firms with powerful CEOs, relying on specifications of the type used earlier to show that rigging is related to CEO power.

Our analysis aside, we were intrigued by the question of what the existence of powerful CEOs means for other executives. Does power filter down to other executives or do other executives suffer at the expense of dominant power? We would encourage Wan to pursue these issues further.

\subsubsection{Evidence from 'Expanded' Sample}

Wan also discusses results that use a substantially expanded sample (increased from about 8,000 to 14,000) in which he estimates the baseline and other regression models. While we do not have Wan's expanded sample, we believe that the expanded sample tends to support the findings of MNS. In particular, Wan reproduces the results in the paper in Table A.1. It is shown that both $\operatorname{Max}$ and $\operatorname{Max} *$ Power are significantly related to total CEO compensation. It is interesting that despite the potentially similar multicollinearity issues between Max and Max* Power, including both of them in the same regression results in Max * Power being statistically significant. Though these results are supportive of MNS, one should, however, interpret these results with caution given the multicollinearity issues we have discussed extensively.

14 This is not to argue that rigging should always occur in firms with powerful CEOs. That would, also depend on the bargaining between the CEO and senior management. 


\begin{tabular}{|c|c|c|c|c|c|c|}
\hline & \multicolumn{6}{|c|}{ Dependent Variable: $\operatorname{Ln}(T D C)$} \\
\hline & \multicolumn{3}{|c|}{ Firm FE } & \multicolumn{3}{|c|}{ Executive FE } \\
\hline & (1) & $(2)$ & (3) & (4) & (5) & (6) \\
\hline $\begin{array}{l}\text { Max } * \text { Power } \\
\quad \text { Index }_{i t}\end{array}$ & $\begin{array}{r}0.0178^{* * *} \\
{[0.00657]}\end{array}$ & & & $\begin{array}{r}0.0123^{* * *} \\
{[0.00451]}\end{array}$ & & \\
\hline $\begin{array}{l}\text { Max* } \\
\quad \text { Insider } \%_{i t}\end{array}$ & & $\begin{array}{r}0.131^{* * *} \\
{[0.0476]}\end{array}$ & & & $\begin{array}{l}0.0928^{* * *} \\
{[0.0288]}\end{array}$ & \\
\hline $\begin{array}{l}\text { Max }^{* \%} \% \\
\quad \text { Appointed }_{i t}\end{array}$ & & & $\begin{array}{c}0.0329 \\
{[0.0208]}\end{array}$ & & & $\begin{array}{c}0.0193 \\
{[0.0137]}\end{array}$ \\
\hline Observations & 33656 & 24891 & 23613 & 33656 & 17757 & 16874 \\
\hline R-squared & 0.164 & 0.138 & 0.136 & 0.231 & 0.366 & 0.341 \\
\hline $\begin{array}{l}\text { Time \& } \\
\text { Firm F.E. }\end{array}$ & Yes & Yes & Yes & Yes & Yes & Yes \\
\hline Other Controls & Yes & Yes & Yes & Yes & Yes & Yes \\
\hline
\end{tabular}

Note: ${ }^{* * *},{ }^{* *}$ and ${ }^{*}$ denote significance at $1 \%, 5 \%$ and $10 \%$ respectively.

Table 9. Rigging by non-CEOs executives.

Description: In this table we report the estimates from the regression that uses the natural log of the total compensation of the non-CEO executives as the dependent variable. We present estimates from firm fixed effects (columns 1-3) and executive fixed effects (columns 4-6) specifications. The innovation in the table is the focus on non-CEOs. We iteratively employ power measures - PowerIndex, Insider\% and \%Appointed - in a lagged performance fixed effects with controls as in MNS. Robust standard errors are in parentheses. Data are for the period 1992 to 2003.

Interpretation: We show that the evidence Wan presents is stronger in firms with powerful CEOs, relying on specifications of the type used earlier to show that rigging is related to CEO power.

\section{Conclusion}

Wan (2013) argues that the statistical inferences in our Journal of Finance (2011) paper are not robust as we do not prove that it is powerful CEOs that rig incentive contracts. In this article we argue that Wan's critique is not supported theoretically or empirically.

Wan makes the theoretical claim that the rigging results are consistent with ex-post optimal re-contracting. However, optimal re-contracting cannot explain the loss in firm value from contract switching we show in the paper. Nor do we know of a theory that would predict that ex-post realignment could be tested using our contract switching term in the wage function, like Wan does.

On the empirical front, Wan's critique has at least three serious flaws. First, his standardized performance measures — different than ours — result in 
accounting returns being 14 percentage points higher than stock returns. Consequently, switching between measures, necessary for identification, is infrequent and outlier-based, not surprisingly delivering regression estimates differing from ours. Second, Wan interprets selectively among insignificant coefficients to make his claims. Third, regardless of interpretation, basic mathematics casts doubt on the premise of his estimation strategy.

Wan makes one valid point: our original work could have provided more extensive cross sectional empirical support for our rigging claims. We take this opportunity to present new cross sectional (between firm) evidence and conclude even more strongly that powerful CEOs sway boards to load their incentive pay on more favorably performing measures.

\section{Appendix A}

Appendix Table 1 presents results from our earlier draft where we had used both Max and Max * Power in specifications with several measures of power. In two of the three rounds in the refereeing process, as well as in all our earlier drafts, we had included both Max and Max*Power in specifications. In the later rounds, the referee and editors suggested that we change the definition of two of our three power measures (Power Index Old-draft and $\%$ Appointed $^{\text {Old-draft }}$ to Power Index ${ }^{J F}$ and \% Appointed ${ }^{J F}$ ) and drop one measure (Centrality ${ }^{\text {Old-draft }}$ based on Bebchuk et al. (2011)), while sticking to the theoretically-driven wage specification. Panel A reports results with all these variables. For most measures, Max*Power is significant, and the positive significance of Max is entirely absorbed by Max*Power variables. For two of the three power measures in the final version of the paper, inclusion of Max washes out the significance of Max*Power. Notably, there is no specification where we find Max is significant and positive while Max* Power is not.

In Panel B, we present the results from an older version where we had included all these variables in the same specification. Again, the significance of Max is entirely absorbed by Max * Power variables.

It is important to reiterate that the reduction in the significance of $\operatorname{Max} *$ Power when Max is introduced does not imply that rigging is not driven by CEOs with power. As we have already explained in detail, it is difficult to identify rigging and its drivers with both Max and Max *Power in the estimation, especially for certain power variables, on account of the very 


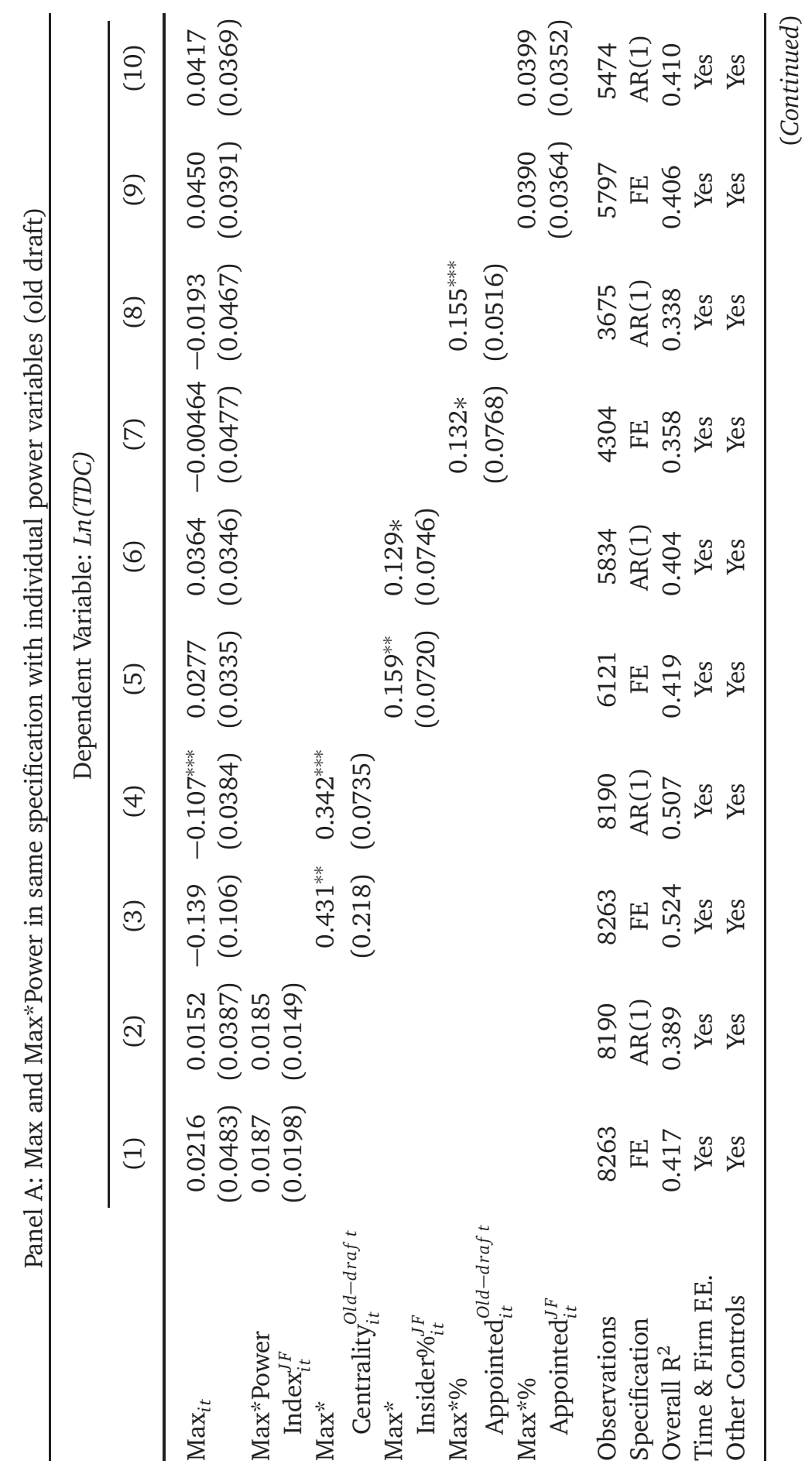




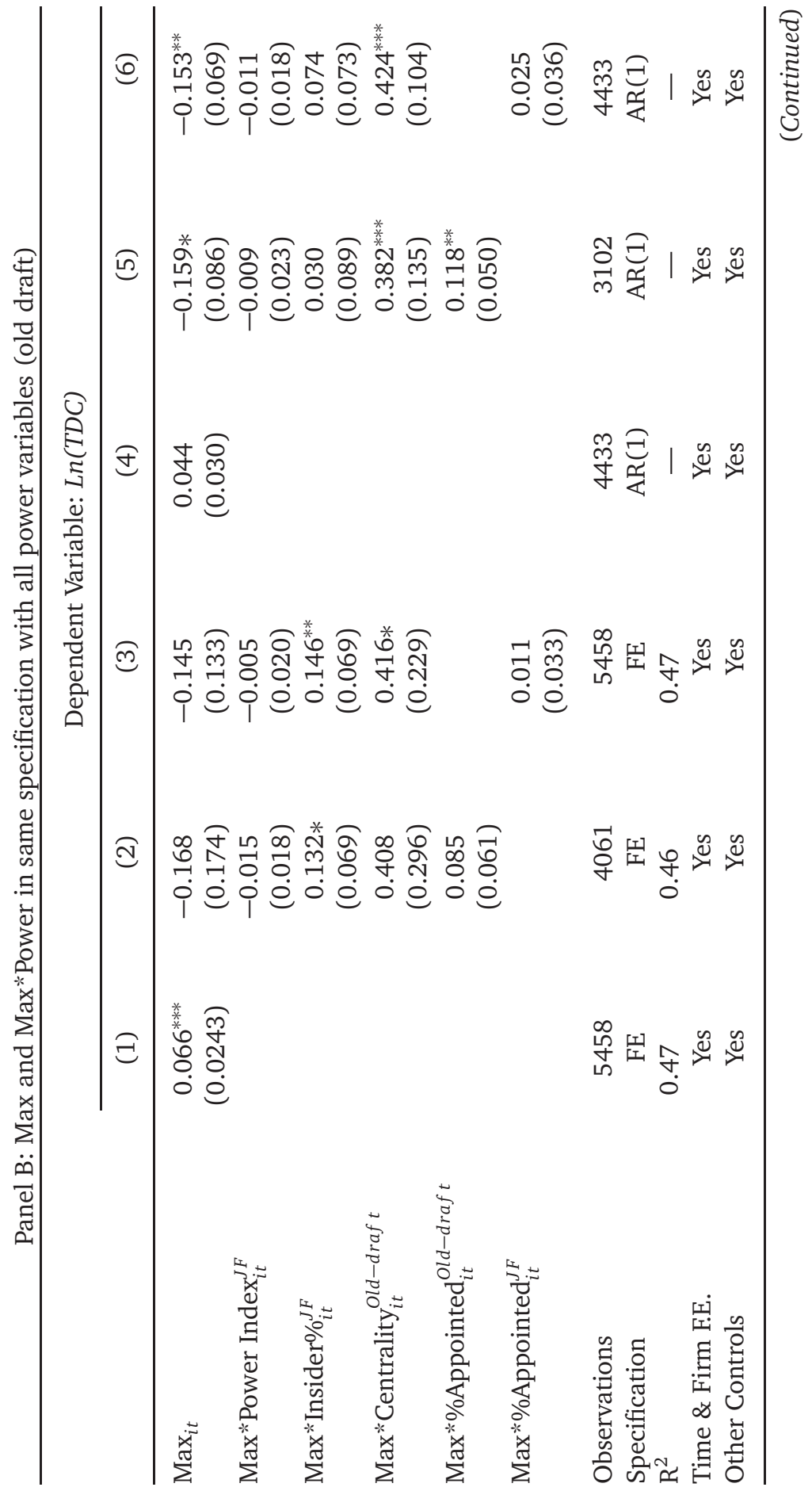




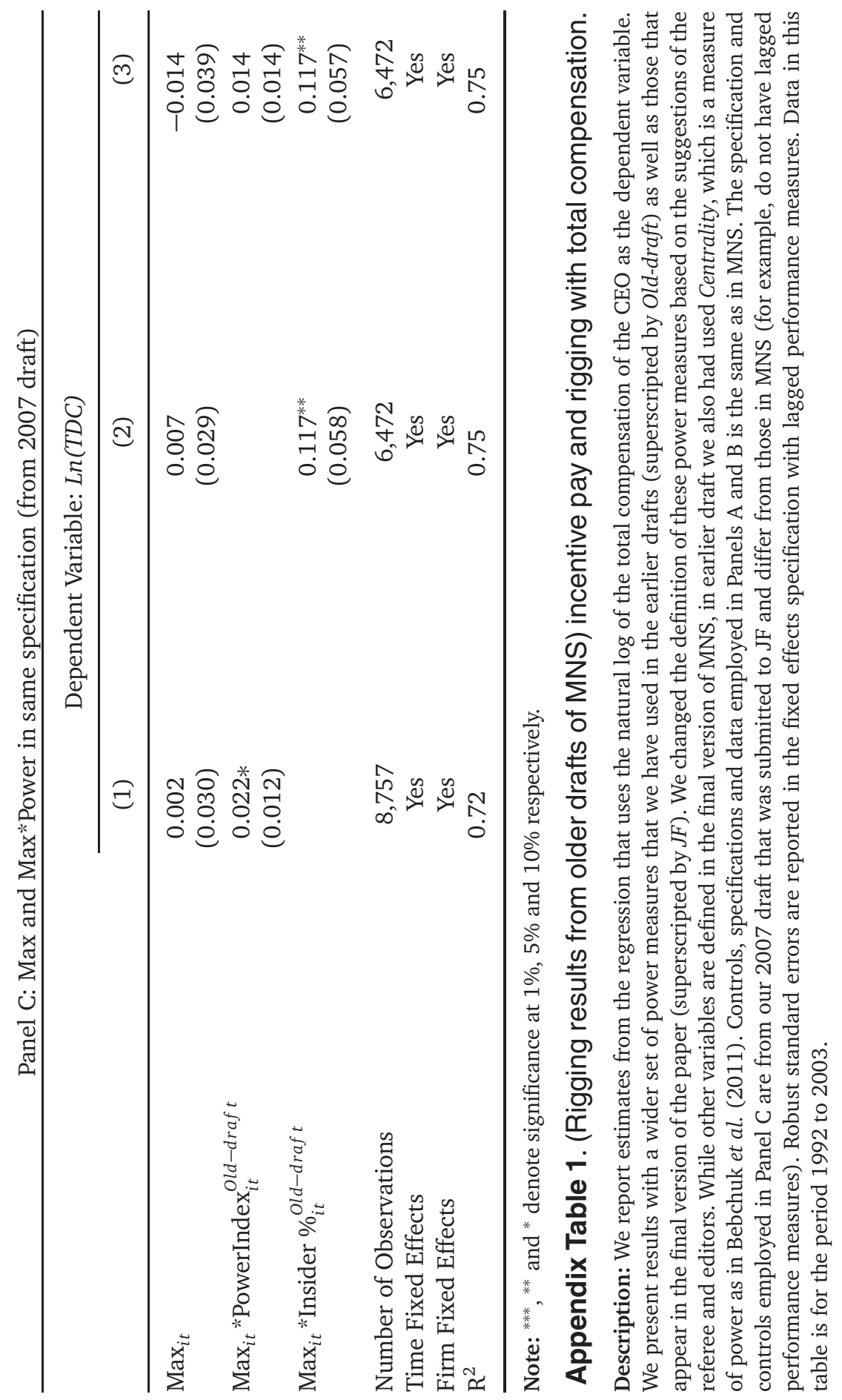


high multicollinearity between these terms. This is the reason that introduction of both these variables blows up the standard errors and mechanically decreases the statistical significance of both these variables, something that Wan ignores. Our conclusion from tests that account for this statistical problem is that the rigging effect in MNS is driven by firms where CEOs have power.

Having said that, our Appendix Table 1 (Panel A) does show that we can identify significant rigging ( $M a x *$ Power) for our \%Insider measure of power. The reason is that \%Insider is a more continuous variable than both PowerIndex (a 3-valued variable) and \%Appointed (a variable with more weights at the 0 and 1 endpoints). It is a well known statistical property that estimates using a continuous variables have more statistical power, in the sense of being able to reject the null hypothesis when it is in fact false (see, MacCallum et al. (2002). This argument for identification using \% Insider also holds for the Centrality ${ }^{\text {Old-draft }}$ power measure that we had used in our earlier version power measure.

It is also worth noting that our results using \%Insider as a power measure differ from those reported in Wan (2013). We do not know why this difference exists. Wan does not report the AR(1) specification so we cannot compare those results. We also note that these results with \%Insider and using both Max and Max * Power in the specification have existed in our paper from versions from five years ago (see Panel C for a Table from our 2007 draft that was submitted to JF).

In Appendix Table 2, we reproduce another table from an older draft of the paper (2007 draft that was submitted to JF). Here, we had demonstrated that the shift towards the measures that are ex-post more favorable occurred for both stock and accounting returns. Note that this specification, again, uses both Max and Max* Power using \%Insider measure of power.

\section{Appendix B}

In this appendix, we consider an alternative specification for the between estimator. We start from Equation (6), which we repeat here:

$$
\overline{\widetilde{y}}_{i}=\beta_{0}^{p}+\overline{\widetilde{X}}_{i}^{\prime} \beta_{1}^{p}+\left(c_{i}^{p}-\beta_{0}^{p}+\bar{\varepsilon}_{i}\right), \text { for all } i \text { in } p .
$$

Rather than allowing $X$ to capture all covariates, we assume that it does not incorporate performance. Since time variation in performance is the crux of 
Panel A: Fixed Effect Specification (from 2007 draft)

\begin{tabular}{|c|c|c|c|}
\hline & \multicolumn{3}{|c|}{ Dependent Variable: $\operatorname{Ln}(T D C)$} \\
\hline & $\begin{array}{l}\text { (1) } \\
\text { Remove Top Quartile of } \\
\text { Accounting Performers }\end{array}$ & $\begin{array}{c}\text { (2) } \\
\text { Remove Top } \\
\text { Quartile of Stock } \\
\text { Performers }\end{array}$ & $\begin{array}{l}\text { (3) } \\
\text { Remove Stock and } \\
\text { Accounting Both Positive }\end{array}$ \\
\hline Insider $\%{ }_{i t}^{\text {Old }}$-draft & $\begin{array}{c}0.042 \\
(0.067)\end{array}$ & $\begin{array}{c}0.020 \\
(0.070)\end{array}$ & $\begin{array}{c}0.012 \\
(0.074)\end{array}$ \\
\hline $\operatorname{Max}_{i t}$ & $\begin{array}{l}-0.045 \\
(0.057)\end{array}$ & $\begin{array}{c}0.003 \\
(0.047)\end{array}$ & $\begin{array}{c}0.003 \\
(0.044)\end{array}$ \\
\hline $\operatorname{Max}_{i t}{ }^{*}$ Insider $\%_{i t}^{\text {Old }-d r a f t}$ & $\begin{array}{c}0.188^{* * *} \\
(0.063)\end{array}$ & $\begin{array}{c}0.177^{* *} \\
(0.090)\end{array}$ & $\begin{array}{l}0.207^{* * *} \\
(0.069)\end{array}$ \\
\hline Observations & 4,797 & 4,854 & 4,637 \\
\hline Other Controls & Yes & Yes & Yes \\
\hline Time Fixed Effects & Yes & Yes & Yes \\
\hline Firm Fixed Effects & Yes & Yes & Yes \\
\hline $\mathrm{R}^{2}$ & 0.76 & 0.77 & 0.80 \\
\hline
\end{tabular}

Panel B: First Difference Specification (from 2007 draft)

\begin{tabular}{|c|c|c|c|}
\hline & \multicolumn{3}{|c|}{ Dependent Variable: $\operatorname{Ln}(T D C)$} \\
\hline & $\begin{array}{l}\text { (1) } \\
\text { Remove Top Quartile of } \\
\text { Accounting Performers }\end{array}$ & $\begin{array}{c}\text { (2) } \\
\text { Remove Top } \\
\text { Quartile of Stock } \\
\text { Performers }\end{array}$ & $\begin{array}{l}\text { (3) } \\
\text { Remove Stock and } \\
\text { Accounting Both Positive }\end{array}$ \\
\hline Insider ${ }_{i t}^{\text {Old }- \text { draf } t}$ & $\begin{array}{c}-0.033 \\
(0.067)\end{array}$ & $\begin{array}{c}-0.079 \\
(0.068)\end{array}$ & $\begin{array}{c}-0.069 \\
(0.074)\end{array}$ \\
\hline $\operatorname{Max}_{i t}$ & $\begin{array}{c}-0.074 \\
(0.066)\end{array}$ & $\begin{array}{c}-0.048 \\
(0.047)\end{array}$ & $\begin{array}{c}-0.056 \\
(0.045)\end{array}$ \\
\hline $\operatorname{Max}_{i t}{ }^{*}$ Insider $\%{ }_{i t}^{\text {Old }-d r a f t}$ & $\begin{array}{c}0.242^{* *} \\
(0.098)\end{array}$ & $\begin{array}{c}0.252^{* * *} \\
(0.098)\end{array}$ & $\begin{array}{c}0.254^{* * *} \\
(0.094)\end{array}$ \\
\hline Observations & 4,324 & 4,409 & 4,169 \\
\hline $\mathrm{R}^{2}$ & 0.39 & 0.31 & 0.43 \\
\hline Other Controls & Yes & Yes & Yes \\
\hline Time Fixed Effects & Yes & Yes & Yes \\
\hline
\end{tabular}

Note: ${ }^{* * *},{ }^{* *}$ and ${ }^{*}$ denote significance at $1 \%, 5 \%$ and $10 \%$ respectively.

Appendix Table 2. (Results from older draft of MNS) incentive pay and rigging: Performance subsamples.

Description: In this table we report the estimates from fixed effect and first difference specifications estimated in subsamples split based on performance. The dependent variable is constructed using the natural $\log$ of the total compensation of the CEO. In Panel A, we present the fixed effects specification while Panel $\mathrm{B}$, reports results of the first difference specification. The specification and controls in these regressions are different from those in MNS (for example, do not include lagged performance variables) and are from our 2007 version that was submitted to JF. All regressions are estimated with time fixed effects and the standard errors reported in the parenthesis in all the panels are corrected for heteroscedasticity, and cross correlation. Data in this table is for the period 1992 to 2003. 


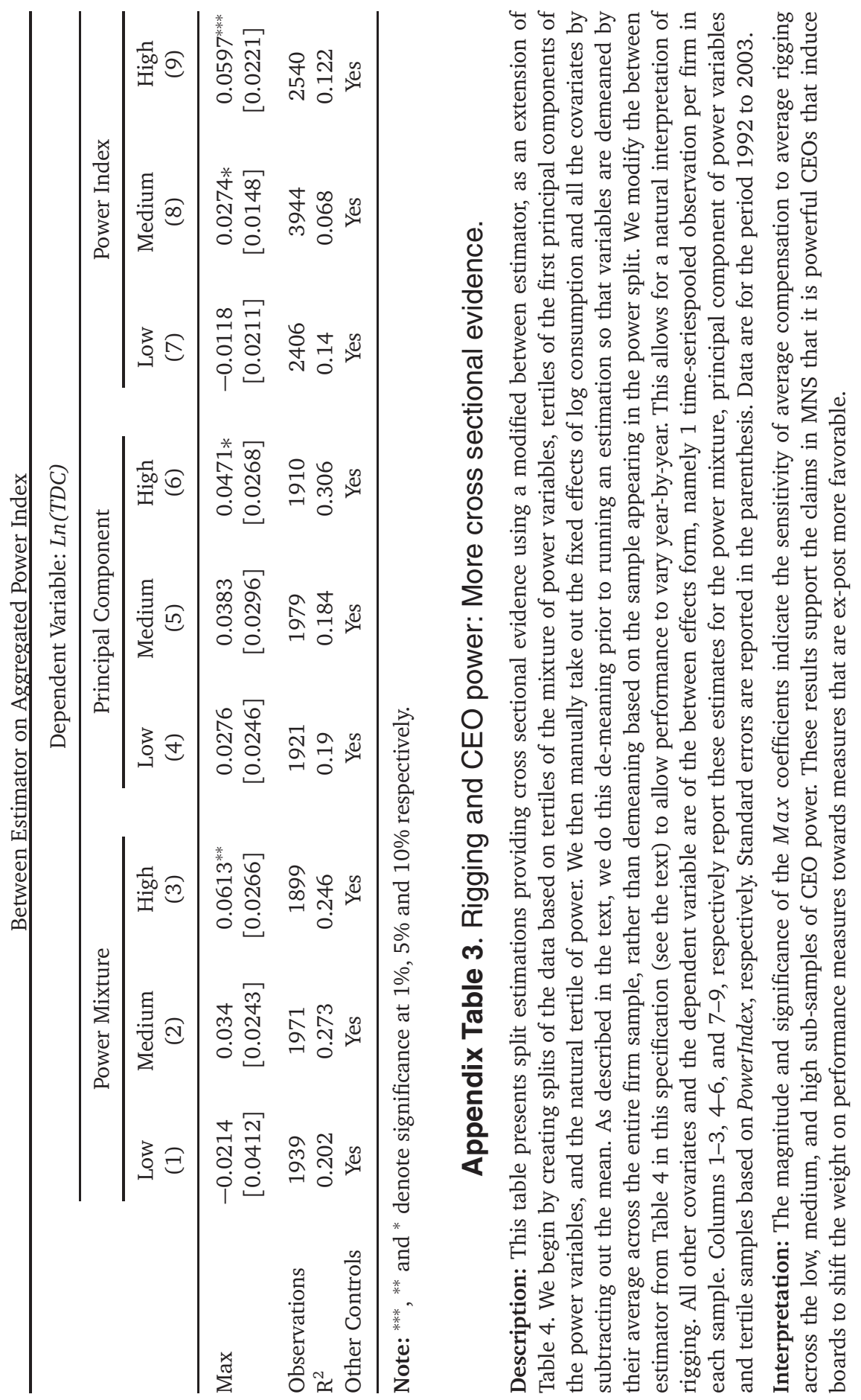


what we are after, we layer in performance and rigging once the between formulation and assumptions have been asserted. In particular, we simply add in the performance variation by year.

$$
\overline{\tilde{y}}_{i}^{p}=\beta_{0}^{p}+\bar{X}_{i}^{p \prime} \beta_{1}^{p}+\beta_{2}^{p} z r o a_{i t}^{p}+\beta_{3}^{p} z t r s_{i t}^{p}+\beta_{4}^{p} \operatorname{Max}_{i t}^{p}+\xi_{i t}^{p} .
$$

We cluster standard errors at the firm level to handle the fact that we have multiple observations with the same averaged dependent variable and covariates other than performance.

Appendix Table 3 present results in power tertiles formed on the basis of power mixture (columns 1-3), principal components based on all power measures (columns 4-6) and the Power Index (columns 7-9). The results confirm our intuition: the sensitivity of compensation to Max is large and significant in the high power subsamples (columns 3, 6, and 9) and is economically and statistically equal to zero in the low power subsamples (columns 1, 4, and 7).

\section{References}

Bebchuk, L., J. Fried, and D. Walker. 2002. "Managerial Power and Rent Extraction in the Design of Executive Compensation." University of Chicago Law Review 69: 751-846.

Bebchuk, L., M. Cremers, and U. Peyer. 2011. “CEO Pay Slice.” Journal of Financial Economics 102(1): 199-221.

Bertrand, M., E. Duflo, and S. Mullainathan. 2004. "How Much Should we Trust Difference in Differences?" Quarterly Journal of Economics 119(1): 249-275.

Cameron, A. C. and P. K. Trivedi. 2005. Microeconometrics. Cambridge University Press, New York.

MacCallum, R. C., S. Zhang, K. J. Preacher, and D. D. Rucker. 2002. "On the Practice of Dichotomization of Quantitative Variables." Psychological Methods 7(1): 19-40.

Wan, K. 2013. "Incentive Contract are not Rigged by Powerful CEOs." Working paper.

Wooldridge, J. M. 2002. Econometric Analysis of Cross Section and Panel Data. MIT Press, Boston. 